\title{
Simulation of STEM-EELS Including Diffraction and Solid-State Effects I: Mixed Dynamic Form Factor beyond the Dipole Approximation
}

\author{
M. P. Prange,* M. P. Oxley,* S. J. Pennycook, ** and S. T. Pantelides* \\ * Department of Physics and Astronomy, Vanderbilt University, Nashville, TN 37235 \\ ** Materials Science and Technology Division, Oak Ridge National Laboratory, Oak Ridge, TN \\ 37831
}

Continuing advances in scanning transmission electron microscopy (STEM), including aberrationcorrection and enhanced stability, have made possible the collection of atomic resolution core-edge electron energy loss spectra (EELS). The dependence of the energy-loss spectrum on the probe position within the unit cell contains information about the spatial distribution and nature of the electronic excitations. Interpretation of such experiments, however, requires simulation to relate the strength of the EELS signal to the electronic structure and dynamics of the sample electrons. Image simulation methods used for STEM typically employ an atomic model for the inelastic scattering, and the simulated STEM-EELS images are smooth in energy-loss, missing the fine structure due to the solid-state environment of the inelastic scattering events. Hence, such simulations are usually limited to chemical maps in which the signal from a given species is integrated over an energy window, and all spectral information except atomic number is discarded. On the other hand, since EELS and x-ray absorption spectroscopy (XAS) probe the same electronic excitations, EELS experiments are frequently interpreted in terms of simulations originally developed for XAS and based on the calculation of the dynamic form factor (DFF) of the sample which is closely related to the (macroscopic) inverse dielectric function. Comparison of such simulations to measurements can be theoretically justified when the probe beam is broad so that many unit cells are interrogated simultaneously and diffraction of the probe beam by the sample can be neglected. Both of these conditions are severely violated in atomic-resolution STEM.

We present a theoretical method to calculate a generalization of the DFF, called the mixed dynamic form factor (MDFF), which is closely related to the microscopic inverse dielectric function and contains enough information to predict the STEM-EELS image. The method is based on plane-wave density-functional-theory (DFT) calculations of the electronic structure using the projectoraugmented wave (PAW) method which allows the recovery of the all-electron Kohn-Sham wave functions from a plane-wave calculation. These wave functions serve as input for new codes which compute, for each state in the DFT calculations, matrix elements $M_{f i}(\vec{q})=\left\langle i\left|e^{i \vec{q} \cdot \vec{r}}\right| f\right\rangle$ between the DFT states $|f\rangle$, calculated in the presence of a core hole, and Bloch sums $|i\rangle$ of core level wave functions. These matrix elements and the calculated band structure can be used to construct the MDFF via

$$
S\left(\vec{q}, \vec{q}^{\prime}, \omega\right)=\sum_{f, i}=M_{f i}(\vec{q}) M_{f i}^{*}\left(\vec{q}^{\prime}\right) \delta\left(E_{f i}-\hbar \omega\right)
$$

In a sequel to this talk, Mark Oxley will show how these ingredients are used in a STEM-EELS simulation. In the current talk we present applications of the matrix elements to inelastic x-ray scattering experiments. In these calculations the usual dipole approximation is avoided so that the results are valid at any momentum transfer, even outside the Brillouin zone. We present calculations 
of the dipole forbidden s-s transitions that comprise the $\mathrm{Na}$ K-edge exciton in $\mathrm{NaCl}$ and compare them to X-ray measurements [1],[2].

References

[1] K. P. Nagle1, G. T. Seidler, E. L. Shirley, T. T. Fister, J. A. Bradley, and F. C. Brown, PRB 80, 045105 (2009).

[2] This research was supported by DOE grant DE- F002-09ER46554 ( MPP, MPO, STP), and the Office of Basic Energy Sciences, Materials Sciences and Engineering Division, U.S. Department of Energy (SJP).

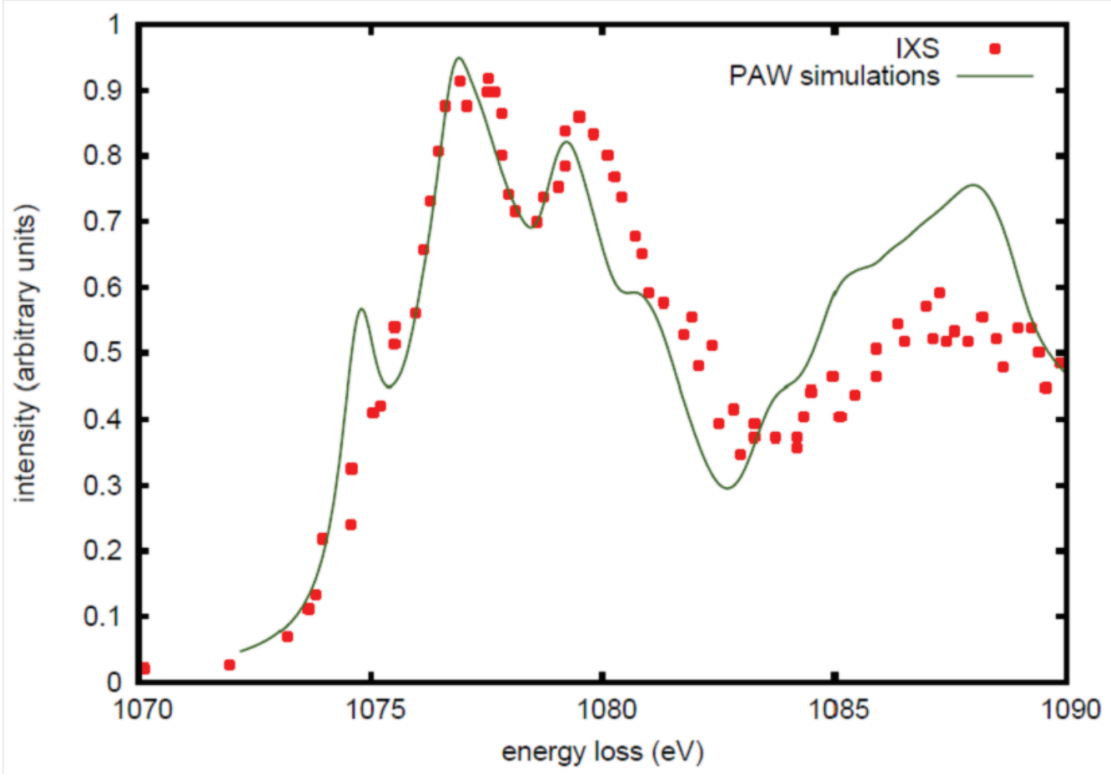

FIG. 1 Sodium K-edge IXS spectra in $\mathrm{NaCl}$ from [1] (red dots) compared to our PAW calculations of the DFF.

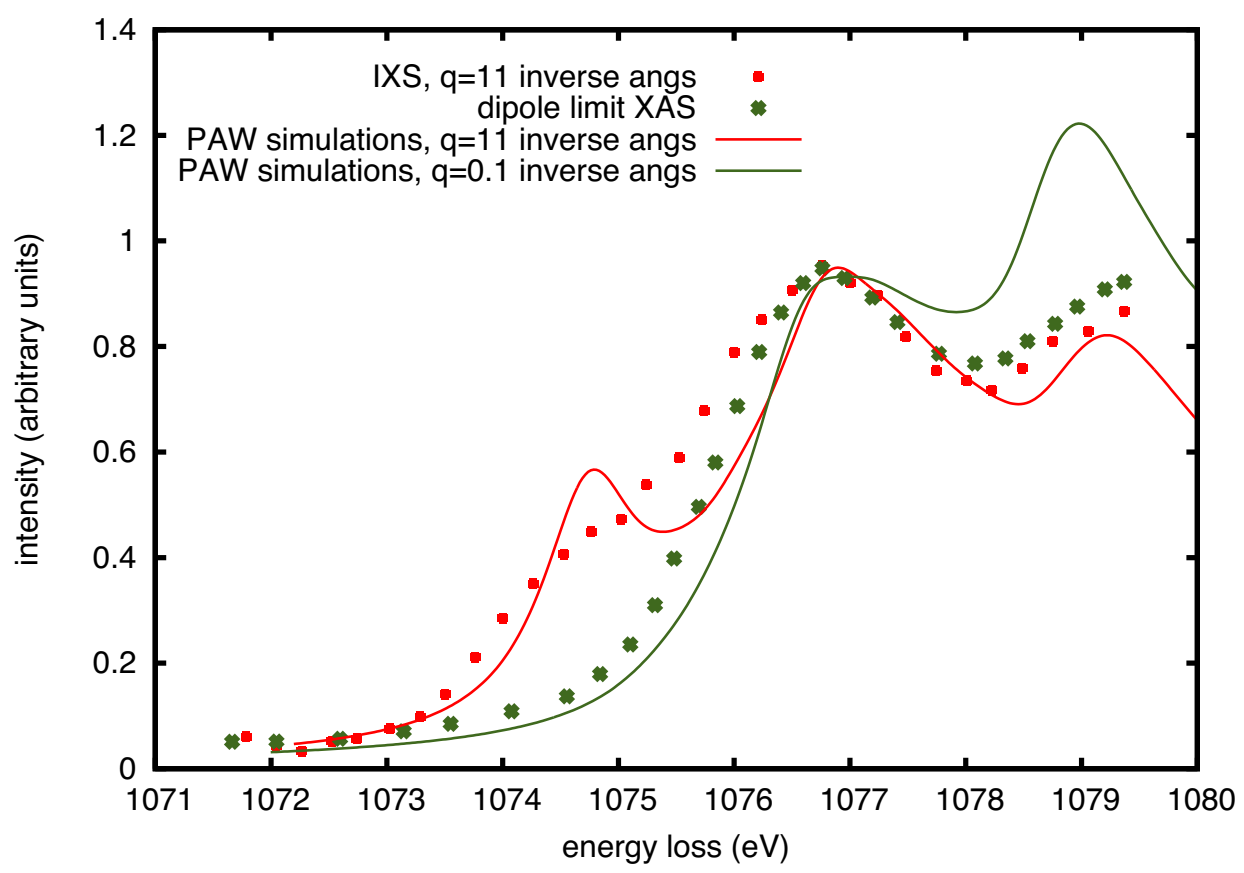

FIG. 2 Pre-edge region of the Sodium $\mathrm{K}$ edge as measured by high impulse IXS [1] (red dots) and low impulse XAS [1] (green dots) compared to simulations at high and low momentum transfers. The extra oscillator strength shown by the IXS measurement between $1074 \mathrm{eV}$ and $1076 \mathrm{eV}$ is due to dipole forbidden s-s transitions. 